\title{
La pandemia de la COVID-19 y su impacto en la educación superior
}

\section{The covid 19 pandemic and its impact on higher education}

\author{
Aníbal Valentín Díaz-Lazo 1,a \\ https://orcid.org/0000-0002-8340-7898
}

\section{Citar como}

Diaz-Lazo A. La pandemia del COVID 19 y su impacto en la educación superior. Desafíos. 2020; 11(1): 09-10. https://doi.org/10.37711/ desafios.2020.11.1.137

El mundo vive la pandemia de la enfermedad del coronavirus (COVID-19) ocasionado por el coronavirus 2 del síndrome respiratorio agudo severo (SARS-CoV-2), la cual se inició en Wuhan (China) a fines de diciembre del 2019; propagándose progresivamente a todo el mundo y afectando en la actualidad a más de doscientos países (Zhang, 2019; WHO, 2020). En América Latina, las mayores tasas de incidencia por 100 O00 habitantes corresponden a Chile con 1200 casos y a Perú con 730,6 casos (WHO, 2020).

La Organización Mundial de la Salud (OMS) ha anunciado que, en la actualidad, el epicentro se ha desplazado a América Latina, proyectando una alta tasa de mortalidad debida a múltiples factores; uno de ellos la precariedad de sus sistemas de salud, porque no están adecuadamente preparados para enfrentar una pandemia con las características como es la enfermedad de la COVID 19 (Calle, 2020). Es conocido además que existen zonas de elevado riesgo de contagio, como son los hospitales, los mercados, los bancos, el sistema de transporte y, en definitiva, todos los lugares donde existe una alta concentración de personas. También existe evidencia de que las medidas preventivas aceptadas para controlar la propagación de la enfermedad pasan por el distanciamiento entre las personas, el uso de mascarilla, el lavado de manos, el aislamiento social y evitar las aglomeraciones, entre otros.

La situación sanitaria ha generado impactos negativos en diversos sectores, siendo más evidentes en salud, educación y economía. Sin embargo, un análisis del efecto de la enfermedad de la COVID 19 sobre la educación superior, en el corto, mediano y largo plazo, debe pasar por la gobernanza del sistema, la institución, el docente, el estudiante, el personal administrativo, la conectividad a internet y los costos (Giannini, 2020).

En ese panorama se han suspendido las actividades presenciales en las instituciones educativas, se han cancelado las reuniones académicas, cursos, seminarios, congresos, etc; por lo que las universidades se han visto en la necesidad de optar por mecanismos no presenciales, implementando los entornos virtuales y, en aquellas que ya lo tenían funcionando optaron por potenciarlo. En tal sentido se ha promovido la utilización de plataformas en línea o el campus virtual, para continuar facilitando el aprendizaje de los estudiantes a distancia; proporcionando la capacitación sobre la digitalización de las actividades (Crawford, 2020).

La Pandemia por la enfermedad del COVID-19 trajo por tantos cambios y la aplicación de nuevas estrategias de educación a distancia, produciendo innovaciones en las competencias de los docentes en el uso de los entornos virtuales para concretizar el proceso de enseñanza y aprendizaje a través de la utilización de las diversas plataformas (Zoom, Meet, Blackboard, Google Classroom, etc.), la utilización de nuevos instrumentos de evaluación y retroalimentación; así como el empleo de materiales pertinentes para el desarrollo de las sesiones prácticas, tales como los videos u otros medios digitales.

Por consiguiente, es de prever que, en los próximos meses y años, el escenario de la educación superior sufrirá cambios importantes. Por ejemplo, la educación a distancia de tipo sincrónica y asincrónica será

\section{Filiación y grado académico}

'Hospital Regional Docente Clínico Quirúrgico Daniel Alcides Carrión, Huancayo, Perú.

a Doctor en Medicina 
probablemente, parte importante en la formación del profesional del estudiante universitario, en las especialidades declaradas como de formación presencial, en donde los entornos virtuales serán una herramienta complementaria imprescindible.

En definitiva, la creatividad, la innovación y el compromiso de los docentes, permitirán el uso óptimo y adecuado de las plataformas virtuales en el proceso de enseñanza-aprendizaje, así como la predisposición y el interés permanente de los estudiantes a las nuevas formas de la adquisición de conocimientos y competencias. Es de esperar, en todo caso, que dichos aspectos sean pilares para el logro de los objetivos educativos. Sin cambios de paradigmas no hay progreso; por lo que es bueno extraer aquellos aspectos positivos que nos deja la pandemia.

\section{REFERENCIAS BIBLIOGRÁFICAS}

Zhang, L.P., Wang, M., Wang, Y., Zhu, J. y Zhang N. (2019). Focus on a 2019-novel coronavirus (SARS-CoV-2). Future Microbiol. Recuperado de https://doi.org/10.2217/ fmb-2020-0063

World Health Organization. WHO (2020). Director-General's opening remarks at the media briefing on COVID-19: 11 March 2020. Recuperado de 20 https:// www.who.int/dg/speeches/detail/who-directorgeneral-s-opening-remarks-at-the-media-briefing-on-covid-19---11-march-2020

World Health Organization, WHO. (2020). COVID-19 Americas' Regional Dashboard. Geographic Distribution of Cases and Deaths. PAHO COVID 19. Regional HUB. Recuperado de https://who.maps.arcgis.com/apps/ dashboards/efb745c3d88647779beccb91c0e715f9

Calle, M. (2020). América Latina es el "nuevo epicentro" de la pandemia del COVID 19: OMS. Recuperado de https:// www.france24.com/es/20200523-am\%C3\%A9ricalatina-es-el-nuevo-epicentro-de-la-pandemia-delcovid-19-oms

Giannini, S. (2020). COVID 19 y educación superior: de los efectos inmediatos al día después: Análisis de impactos, respuestas políticas y recomendaciones. Recuperado de http://www.iesalc.unesco.org/wp-content/ uploads/2020/05/COVID-19-ES-130520.pdf

Crawford, J., Butler-Henderson, K., Rudolph, J., Malkawi, B., Glowattz, M., Burton, R., et al. (2020). COVID-19: 20 countries' higher education intra-period digital pedagogy responses. Journal of applied learning \& teaching, 3(1), 1-20. Recuperado de https://doi. org/10.37074/jalt.2020.3.1.7

\section{Correspondencia}

Email: andiaz55@hotmail.com 